\title{
Day-night asymmetries of low-energy electrons in Saturn's inner magnetosphere
}

\author{
A. D. DeJong, ${ }^{1}$ J. L. Burch, ${ }^{1}$ J. Goldstein, ${ }^{1}$ A. J. Coates, ${ }^{2}$ and F. Crary ${ }^{1}$ \\ Received 1 March 2011; revised 21 March 2011; accepted 28 March 2011; published 23 April 2011.
}

[1] We examine the day-night asymmetry of near-equatorial low energy (12-100 eV) electron fluxes measured by Cassini from July 1, 2004 through April 1, 2010. This energy range is also known to be associated with interchange injections. The electrons are separated into field-aligned $\left(0^{\circ}\right.$ to $20^{\circ}$ and $160^{\circ}$ to $\left.180^{\circ}\right)$ pitch angles and trapped $\left(70^{\circ}\right.$ to $\left.110^{\circ}\right)$ pitch angles. There is a stronger day-night asymmetry for the trapped than the field-aligned electrons, but both show enhanced energy fluxes on the nightside relative to the dayside. The dayside electron fluxes decrease sharply at an L-shell of 8 , while the nightside electrons exhibit a slow decline in to $\mathrm{L}=5$. Our finding, along with previous research of high energy electrons, shows that this asymmetry is energy independent. This suggests that interchange injections are stronger, and therefore penetrate deeper into the magnetosphere, on the nightside. Citation: DeJong, A. D., J. L. Burch, J. Goldstein, A. J. Coates, and F. Crary (2011), Day-night asymmetries of low-energy electrons in Saturn's inner magnetosphere, Geophys. Res. Lett., 38, L08106, doi:10.1029/ 2011 GL047308.

\section{Introduction}

[2] Using Cassini Plasma Spectrometer (CAPS) data Young et al. [2004], Burch et al. [2005] and Hill et al. [2005] found evidence of a centrifugal interchange instability that causes magnetic flux tubes containing low density, high temperature plasma to replace dense, cold plasma in Saturn's inner magnetosphere. When electrons and ions of an interchange injection are observed together, they disperse in opposite directions in the corotating frame, due to gradient-curvature drifts. The azimuthal separation between these dispersions indicates the age of the interchange injection [Hill et al., 2005]. Young, or local, injections exhibit less dispersion and have a decrease in electron energy flux at lower energies $(<100 \mathrm{eV})$ [Burch et al., 2005]. Older injections tend to have more dispersion in the high energy range $(>1000)$ and no longer possess a flux decrease at lower energies. Chen and Hill [2008] performed a statistical analysis on 429 separate injections/dispersions and found a local-time asymmetry with injections clustered in the pre-noon quadrant. These plasma injections also appear to have a radial dependence, because they are only found between 5 and $10 \mathrm{R}_{S}$ [Burch et al., 2005; Hill et al., 2005; Chen and Hill, 2008]. Müller et al. [2010] analyzed 52 injections, using the Magneto-

\footnotetext{
${ }^{1}$ Southwest Research Institute, San Antonio, Texas, USA.

${ }^{2}$ Mullard Space Science Laboratory, University College London, Dorking, UK.
}

Copyright 2011 by the American Geophysical Union. 0094-8276/11/2011GL047308 spheric Imaging Instrument (MIMI) on Cassini. They traced the injections back to their points of origin and found that the injections occur with a higher frequency in the night and morning sectors.

[3] Carbary et al. [2009] and Paranicas et al. [2010] used Low Energy Magnetospheric Measurement System (LEMMS) MIMI data to perform a statistical study of the electrons fluxes in the inner magnetosphere of Saturn. They found larger electron energy fluxes on the nightside relative to the dayside. Also using MIMI measurements, Krimigis et al. [2007] identified a local time asymmetry in the ring current and pressure. Thus, numerous studies have shown daynight asymmetries in the Saturnian magnetosphere.

[4] DeJong et al. [2010] showed that increases in the energy flux for 12-100 eV electrons are associated with interchange injections. When averaging 3 years of equatorial data, they identified a peak in energy flux for the $12-100 \mathrm{eV}$ electrons at approximately $8 \mathrm{R}_{S}$. They then separated the electrons by pitch angle into trapped and field-aligned populations. While both pitch angle populations exhibited an increase in energy flux associated with injections, the fieldaligned electrons showed larger increases relative to the trapped electrons. Therefore, the authors concluded that the field-aligned electrons in the $12-100 \mathrm{eV}$ are more closely linked to the interchange processes than the trapped electrons. They believe that the field-aligned electrons are driven into the magnetosphere from the ionosphere by the field-aligned currents that are associated with interchange injections. DeJong et al. [2010, Figure 9a] indicated that there may be a nightside-dayside asymmetry in the $12-100 \mathrm{eV}$ electrons with the nightside electrons penetrating deeper into the magnetosphere. In this investigation, we expand on the findings of DeJong et al. [2010] and others by investigating possible dayside and nightside asymmetries in electron energy fluxes measured by CAPS ELS (Electron Spectrometer) in the Saturnian magnetosphere.

\section{Data Selection and Analysis}

[5] While the CAPS ELS instrument on Cassini can measure fluxes of electrons ranging from $0.5 \mathrm{eV}$ to $28,000 \mathrm{eV}$, only the $12-100 \mathrm{eV}$ electrons are investigated. The lower limit of $12 \mathrm{eV}$ is imposed because $12.6 \mathrm{eV}$ is the ionization energy of water in the gaseous state [Johnson, 1990] and because electrons below this range are most likely photoelectrons emitted by the Cassini spacecraft. The upper limit of $100 \mathrm{eV}$ is employed since it is the approximate boundary between the energy ranges of the bimodal electrons identified by Sittler et al. [1983] and Young et al. [2005] in Saturn's magnetosphere.

[6] The CAPS ELS data in this study were taken from July 1, 2004 to April 1, 2010 when Cassini was located 


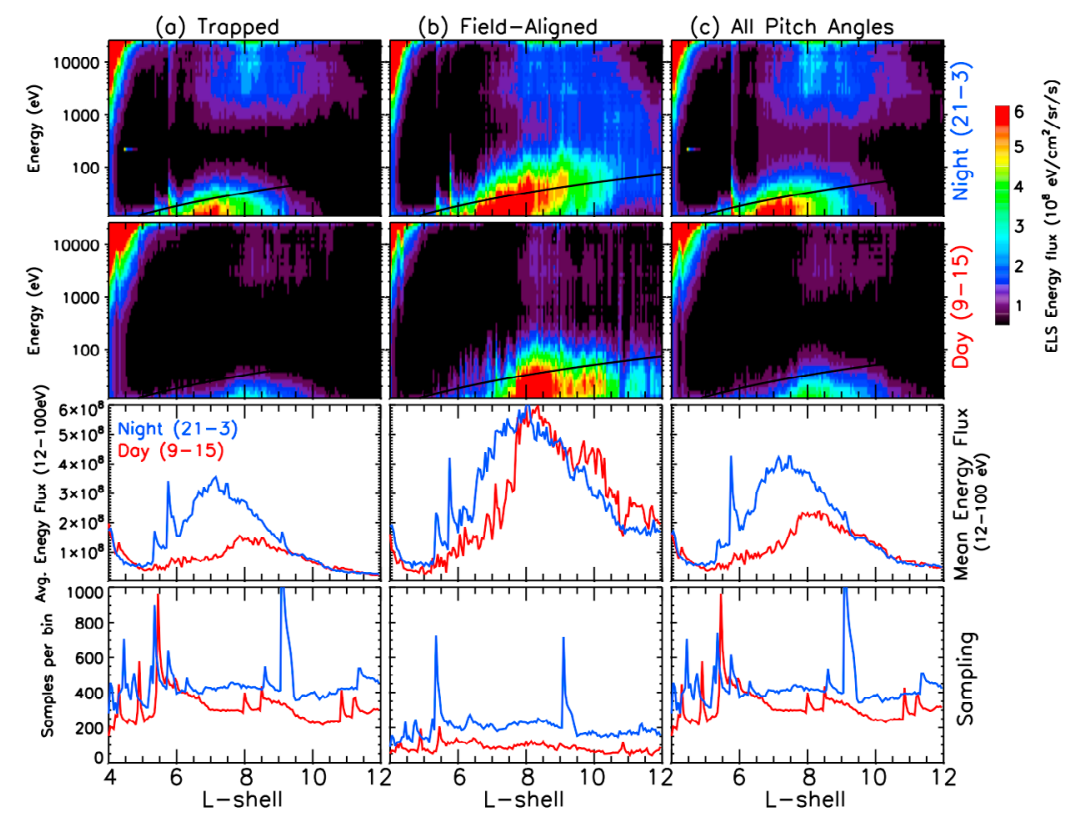

Figure 1. Energy ( $12 \mathrm{eV}$ to $26040 \mathrm{eV}$ ) versus L-shell (4 to 12) spectrogram for average fluxes of electrons with (a) pitch angle between $70^{\circ}-110^{\circ}$, trapped electrons (b) field-aligned electrons, pitch angles less than $20^{\circ}$ and great than $160^{\circ}$ (c) all electrons, regardless of pitch angle. (top) Averaged over the night side (21-3 UT), (middle top) averaged over the dayside (9-15 UT), (middle bottom) the average energy fluxes for electrons with energies between 12 and $100 \mathrm{eV}$ from the spectrogram above, red $=$ dayside, blue $=$ nightside. (bottom) Plots of the number of samples per bin $(20$ bins per L-shell $)$. All data plotted are from CAPS ELS from 1942004 to 1002010 when Cassini is at latitudes from $+10^{\circ}$ to $-10^{\circ}$.

between $\pm 10^{\circ}$ latitude. The electrons are separated into field-aligned $\left(0^{\circ}\right.$ to $20^{\circ}$ and $160^{\circ}$ to $\left.180^{\circ}\right)$ pitch angles, trapped $\left(70^{\circ}\right.$ to $\left.110^{\circ}\right)$ pitch angles, and all pitch angles (an average of all 8 detectors on ELS). Next, the data sets are binned in $0.05 \mathrm{~L}$-shell increments (20 bins per L-shell) and averaged. Figure 1 shows the binned spectrograms of this averaging for trapped, field-aligned, and all pitch angles for the nightside (21-3 LT) in the first row and the dayside $(9-15$ LT) in the second row. The dawn and dusk regions are not included due to lack of coverage by the Cassini spacecraft. The color bar to the right of the plot shows electron energy flux with black indicating that fluxes are less than $0.5 \times 10^{8} \mathrm{eV} / \mathrm{cm}^{2} / \mathrm{sr} / \mathrm{s}$ and red peaking out at $6.0 \times$ $10^{8} \mathrm{eV} / \mathrm{cm}^{2} / \mathrm{sr} / \mathrm{s}$. The red peak in the upper left corner of each spectrogram are penetrating electrons from the radiation belts.

[7] Figures 1 (top) and 1 (middle top) show a clear separation between the high and low energy electron fluxes at approximately $100 \mathrm{eV}$ on both the dayside and the nightside, supporting the collective findings by Sittler et al. [1983], Young et al. [2005], and Rymer et al. [2007]. The higher energy electrons $(>100 \mathrm{eV})$ possess a larger energy flux on the nightside at all pitch angles. Since the electrons in this energy range are directly related to interchange injections [Burch et al., 2005; DeJong et al., 2010], it appears that, when averaging the equatorial CAPS ELS data from mid 2004 to early 2010, there is a preference for interchange injections to occur on the nightside.

[8] Figure 1 (middle bottom) is the average energy flux for $12-100 \mathrm{eV}$ electrons for both the dayside (red) and the nightside (blue). By inspecting these line plots, along with the spectrograms in the top two rows, we find asymmetries between the dayside and nightside of the inner magnetosphere of Saturn. Both the trapped electrons (Figure 1a) and all pitch angle electrons (Figure 1c) show a large difference in the average energy flux of $12-100 \mathrm{eV}$ electrons between dayside and nightside. By contrast, both the dayside and nightside field-aligned electrons (Figure 1b) have approximately the same average energy flux.

[9] The day-night asymmetry in the penetration of the 12-100 eV electrons into the inner magnetosphere represents one of the key results from Figure 1. There is a sharp decrease in the average energy flux for these electrons just inside of $\mathrm{L}=8$ on the dayside. The decrease also corresponds with a reduction in the high energy $(>100 \mathrm{eV})$ electron fluxes a $\mathrm{L}=8$. In contrast, on the nightside the high energy electrons penetrate into $\mathrm{L} \sim 6.5$, while the low energy electron fluxes slowly decrease in until $\mathrm{L}=5$. This asymmetry occurs in all three pitch angle categories, and can be seen in the line plots of $12-100 \mathrm{eV}$ electrons (third row).

[10] Our results are similar to those of Paranicas et al. [2010] and Carbary et al. [2009], who both used LEMMS electron data. Paranicas et al. [2010] found that trapped electrons in the $41-60 \mathrm{keV}$ range show the same asymmetry as our electron fluxes. Their dayside electrons had less energy flux and only penetrated in to $\mathrm{L}=8$, while their nightside electrons extended inward to $\mathrm{L}=5.5$. Similarly, Carbary et al. [2009] examined higher energy (110-365 keV) electrons and found penetration to $\mathrm{L}=8$ on the dayside and to $\mathrm{L}=5$ on the nightside. These findings along with ours, suggests an energy independent local-time asymmetry in Saturn's magnetosphere that does not allow the electrons to penetrate any deeper than $\mathrm{L}=8$ on the dayside. These penetration differences could also be explained by enhanced 


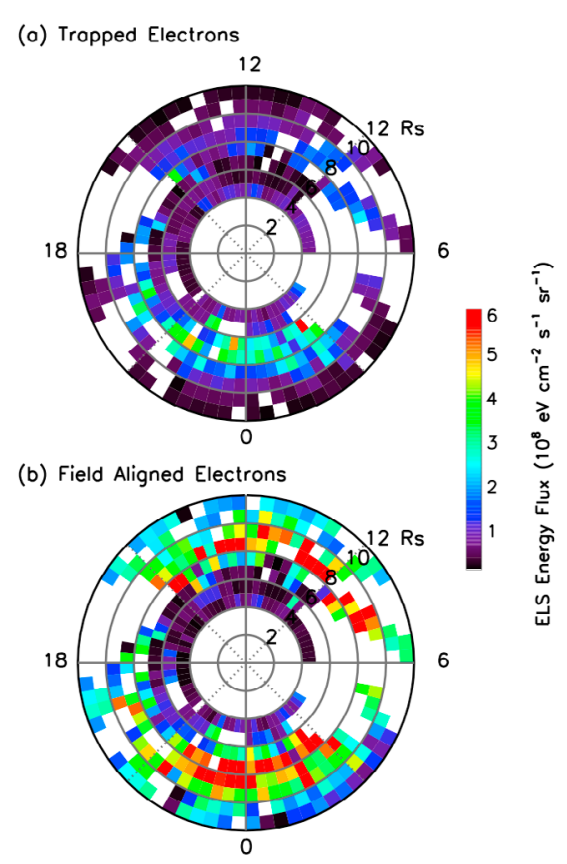

Figure 2. Local time plot of the average energy flux for $12-100 \mathrm{eV}$ electrons (a) trapped electrons $70^{\circ}-110^{\circ}$ pitch angles and (b) field aligned pitch angles less than $20^{\circ}$ and great than $160^{\circ}$. Both plots use the color scale on the right. This is the same data used in Figures 1a (top), 1a (middle top), $1 \mathrm{~b}$ (top), and $1 \mathrm{~b}$ (middle top).

plasma pressure on the dayside relative to the nightside magnetosphere [Krimigis et al., 2007], where the lower pressures on the nightside allow stronger injections to move deeper into the magnetosphere.

[11] Figure 1 (bottom) shows the number of samples per bin (20 bins per $1 R_{S}$ ) for the dayside (red) and nightside (blue). Note that there is higher sampling by CAPS ELS on the nightside than the dayside for all three pitch angle categories. Similarly, there are almost 3 times more trapped electrons than field-aligned electrons. However, since we are investigating the averages of each pitch angle population, the comparison between trapped and field-aligned electrons, and the comparison between the dayside and nightside electrons remains unbiased. On the other hand, when examining the average electron fluxes all pitch angles (Figure 1c), the trapped electrons dominate and cause the spectrograms and plots to appear more like the trapped electrons.

[12] Figure 2 is an alternative approach to visualizing the distribution of the average of the energy fluxes for $12-100 \mathrm{eV}$ electrons. The energy fluxes for the $12-100 \mathrm{eV}$ electrons have been averaged and binned by 1 hour of local time and 1 $\mathrm{R}_{S}$. While, this method possesses less resolution than that of Figure 1, similar patterns can still be seen (note that white pixels indicates no data). The trapped electron fluxes once again exhibit a strong asymmetry, with the nightside having a higher average energy flux than the dayside. While there is also a similar asymmetry for the $12-100 \mathrm{eV}$ field-aligned electrons, it is not as apparent. Both plots indicate a peak in average energy flux occurs at $8 \mathrm{R}_{S}$, as those of DeJong et al. [2010].

[13] The sampling for each bin in Figure 2 is shown in Figure 3 in order to ensure that the local time dependence is not just an artifact of sampling. The correlation between the trapped plots (Figures 2a and 3a) and the field-aligned plots (Figures $2 \mathrm{~b}$ and $3 \mathrm{~b}$ ) are $8.4 \%$ and $10.9 \%$ respectively. These results indicate that less than $11 \%$ of the local time dependence of the electrons can be accounted for by the sampling distribution.

\section{Discussion and Conclusions}

[14] DeJong et al. [2010] showed that electron energy fluxes between 12 to $100 \mathrm{eV}$ inside of $12 \mathrm{R}_{S}$ are related to interchange injections. In this study, we reveal a local time dependence of these electrons and therefore a day-night asymmetry in interchange injections. The energy flux for these cool electrons is intensified on the nightside for trapped electrons, while field-aligned electrons show very little local time variation in the amount of their average energy flux. Both trapped and field-aligned electron energy fluxes exhibit a sharp decrease at $\mathrm{L}=8$ on the dayside. The nightside electrons, on the other hand, slowly decline in average energy flux until $L=5$. This suggests a mechanism in Saturn's magnetosphere that keeps injections from progressing inward of $\mathrm{L}=8$ on the dayside.

[15] Our results, combined with those of Paranicas et al. [2010] and Carbary et al. [2009], demonstrate that the local time differences in penetration of electrons into the inner magnetosphere of Saturn appears to be independent of energy. Thus, it seems unlikely that this penetration asymmetry is due solely to differences between the dayside and nightside absorption of the electron. If this were the case, we would expect larger differences in the L-shell penetration for different electron energies, characteristic of absorption by neutrals.

[16] One possible explanation for this asymmetry in the electron fluxes is reconnection events on the nightside as

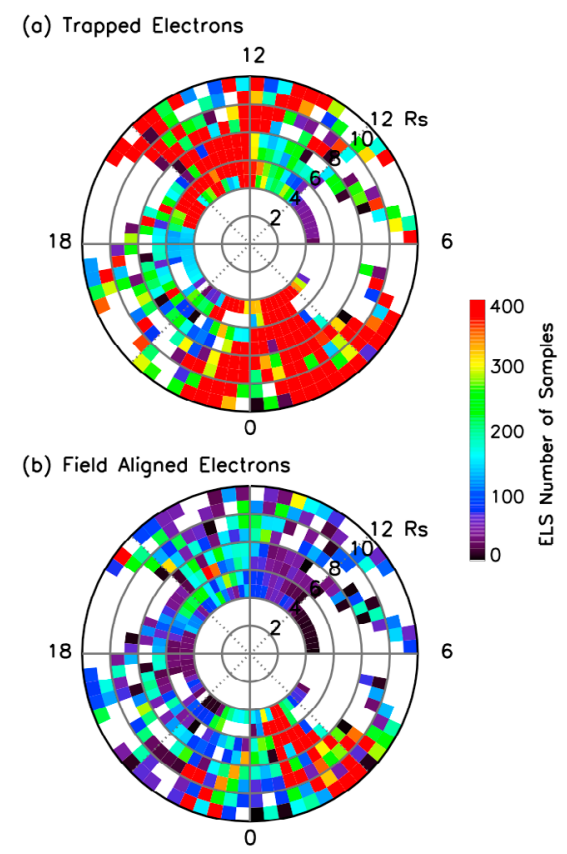

Figure 3. Local time plot of the number of samples in each bin for the plots in Figure 2. Both plots use the color scale on the right. 
noted by Mitchell et al. [2005], Jackman et al. [2007], and Hill et al. [2008]. However, McAndrews et al. [2009] used CAPS data to investigate 35 depleted plasma regions in the tail and conclude that none of their events were created by tail reconnection. Their results indicate that reconnection events in the tail are difficult to measure using CAPS data. Also, from DeJong et al. [2010] and Burch et al. [2005], the electrons both above and below $100 \mathrm{eV}$ as measured by ELS are related to injections; therefore it is more likely that these penetration differences are related to interchange and not reconnection.

[17] Krimigis et al. [2007] noted large differences in the plasma pressure between the dayside and nightside magnetosphere outside of $10 \mathrm{R}_{S}$. Higher pressure on the dayside translates to less pressure difference between the inner and outer magnetosphere in that region. This in turn results in less instabilities, resulting in weaker interchange injections on the dayside. The lower pressure outside of $10 \mathrm{R}_{S}$ on the nightside will increase the strength of the instabilities giving the injections more momentum and allowing them to penetrate deeper into the magnetosphere. While these pressure differences may partial account for local time asymmetries in penetration of the electrons into the inner magnetosphere other mechanisms must be taken into account. If these asymmetries were solely due to pressured differences we should still see electrons inside of $8 \mathrm{R}_{S}$ on the dayside since the nightside injections would rotate to the dayside in approximately 5 hours.

[18] Chen and Hill [2008] found an asymmetry of injection events to favor the pre-noon quadrant, however they excluded young or local injections in their study. Additionally, by tracing injections back to their point of origin, Müller et al. [2010] found hot plasma injections occur primarily in the night and morning sectors between 21 and 9 LT. Furthermore, Mitchell et al. [2005] state that ion acceleration events take place predominately on the night side. Thus, the local time asymmetries that we identify in the CAPS ELS 12-100 eV electron flux data further supports those of Müller et al. [2010] and Mitchell et al. [2005]. However, our analysis only evaluates the mean electron energy flux and does not address the individual injections. Therefore, the next step in understanding the relationship between $12-100 \mathrm{eV}$ electrons and injections is to quantify the correlation between the amount of energy flux and the age of the injections. From our current studies, we anticipate that younger injections will correlate with $12-100 \mathrm{eV}$ electron energy flux.

[19] Acknowledgments. The authors would like to acknowledge Jared Bell for his contribution to the writing and proof reading of this paper. Thanks also goes to M. K. Dougherty, PI of the Cassini magnetic field instrument and MAPSview at the University of Michigan. Work at SwRI was performed under JPL contract NAS703001.

[20] The Editor thanks two anonymous reviewers for their assistance in evaluating this paper.

\section{References}

Burch, J. L., J. Goldstein, T. W. Hill, D. T. Young, F. J. Crary, A. J. Coates, N. André, W. S. Kurth, and E. C. Sittler Jr. (2005), Properties of local plasma injections in Saturn's magnetosphere, Geophys. Res. Lett., 32, L14S02, doi:10.1029/2005GL022611.

Carbary, J. F., D. G. Mitchell, N. Krupp, and S. M. Krimigis (2009), L shell distribution of energetic electrons at Saturn, J. Geophys. Res., 114, A09210, doi:10.1029/2009JA014341.

Chen, Y., and T. W. Hill (2008), Statistical analysis of injection/dispersion events in Saturn's inner magnetosphere, J. Geophys. Res., 113, A07215, doi:10.1029/2008JA013166.

DeJong, A. D., J. L. Burch, J. Goldstein, A. J. Coates, and D. T. Young (2010), Low-energy electrons in Saturn's inner magnetosphere and their role in interchange injections, J. Geophys. Res., 115, A10229, doi:10.1029/ 2010JA015510.

Hill, T. W., A. M. Rymer, J. L. Burch, F. J. Crary, D. T. Young, M. F. Thomsen, D. Delapp, N. André, A. J. Coates, and G. R. Lewis (2005), Evidence for rotationally driven plasma transport in Saturn's magnetosphere, Geophys. Res. Lett., 32, L14S10, doi:10.1029/2005GL022620.

Hill, T. W., et al. (2008), Plasmoids in Saturn's magnetotail, J. Geophys Res., 113, A01214, doi:10.1029/2007JA012626.

Jackman, C. M., C. T. Russell, D. J. Southwood, C. S. Arridge, N. Achilleos, and M. K. Dougherty (2007), Strong rapid dipolarizations in Saturn's magnetotail: In situ evidence of reconnection, Geophys. Res. Lett., 34, L11203, doi:10.1029/2007GL029764.

Johnson, R. E. (1990), Energetic Charged-Particle Interactions With Atmospheres and Surfaces, Springer, Berlin.

Krimigis, S. M., N. Sergis, D. G. Mitchell, D. C. Hamilton, and N. Krupp (2007), A dynamic, rotating ring current around Saturn, Nature, 450, $1050-1053$

McAndrews, H. J., et al. (2009), Plasma in Saturn's nightside magnetosphere and the implications for global circulation, Planet. Space Sci., $57,1714-1722$

Mitchell, D. G., et al. (2005), Energetic ion acceleration in Saturn's magnetotail: Substorms at Saturn?, Geophys. Res. Lett., 32, L20S01, doi:10.1029/2005GL022647.

Müller, A. L., J. Saur, N. Krupp, E. Roussos, B. H. Mauk, A. M. Rymer, D. G. Mitchell, and S. M. Krimigis (2010), Azimuthal plasma flow in the Kronian magnetosphere, J. Geophys. Res., 115, A08203, doi:10.1029/ 2009JA015122.

Paranicas, C., et al. (2010), Transport of energetic electrons into Saturn's inner magnetosphere, J. Geophys. Res., 115, A09214, doi:10.1029/ 2010JA015853.

Rymer, A. M., et al. (2007), Electron sources in Saturn's magnetosphere, J. Geophys. Res., 112, A02201, doi:10.1029/2006JA012017.

Sittler, E. C., Jr., K. W. Ogilvie, and J. D. Scudder (1983), Survey of Low-energy plasma electrons in Saturn's magnetosphere: Voyagers 1 and 2, J. Geophys. Res., 88, 8847-8870.

Young, D. T., et al. (2004), Cassini plasma spectrometer investigation, Space Sci. Rev., 114, 1-112.

Young, D. T., et al. (2005), Composition and dynamics of plasma in Saturn's magnetosphere, Science, 307, 1262-1266.

J. L. Burch, F. Crary, A. D. DeJong, and J. Goldstein, Southwest Research Institute, PO Drawer 28510, San Antonio, TX 78228-0510, USA. (jburch@swri.edu; fcrary@swri.edu; adejong@swri.edu; jgoldstein@ swri.edu)

A. J. Coates, Mullard Space Science Laboratory, University College London, Holmbury St. Mary, Dorking RH5 6NT, UK. (ajc@mssl.ucl.ac.uk) 